\title{
Desempenho de Trichogramma pratissolii Querino \& Zucchi (Hymenoptera, Trichogrammatidae) em ovos de Anagasta kuehniella (Zeller) (Lepidoptera, Pyralidae) sob efeito de Bacillus thuringiensis Berliner
}

\author{
Trichogramma pratissolii Querino \& Zucchi (Hymenoptera, Trichogrammatidae) \\ performance in Anagasta kuehniella (Zeller) (Lepidoptera, Pyralidae) eggs affected \\ by Bacillus thuringiensis Berliner
}

\author{
Dirceu Pratissoli ${ }^{1}$ Ricardo Antonio Polanczyk ${ }^{2}$ Ulysses Rodrigues Vianna ${ }^{3}$ \\ Gilberto Santos Andrade ${ }^{4}$ Regina Gonçalves dos Santos Oliveira ${ }^{5}$
}

RESUMO

Visando estudar o efeito da bactéria entomopatogênica Bacillus thuringiensis (Bt) sobre fêmeas adultas de Trichogramma pratissolii e sua progênie, este trabalho foi realizado com diferentes isolados de Bt e Bt kurstaki misturados ao alimento fornecido para o parasitóide antes de parasitar ovos de Anagasta kuehniella. Para tanto, suspensões de seis isolados de Bt E-3, E-10, E-15, E-16, E-19, E-20 e o Bt kurstaki foram misturados em gotícula de mel (1:1), como fonte de alimento e mel puro como testemunha, e, em seguida, foram oferecidos simultaneamente cartelas com ovos do hospedeiro para o parasitismo. Foram utilizadas 20 repetições por tratamento. O experimento foi mantido em câmara climatizada a $25 \pm 1{ }^{\circ} \mathrm{C}$, UR $70 \pm 10 \%$ e fotofase de $14 \mathrm{~h}$. Foram avaliados os parasitismos diários, totais e acumulados; sobrevivência; emergência e razão sexual da progênie. Os tratamentos não afetaram o parasitismo, razão sexual e a longevidade, porém em alguns tratamentos (E-3, E-10, E-16 e E-20) foi observado efeito indireto sobre a emergência da progênie, o que implicaria a necessidade de mais liberações massais do parasitóide para alcançar os resultados esperados. Por outro lado, a aceleração do parasitismo verificada em todos os tratamentos sugere que adultos de T. pratissolii, quando submetidos à pressão de algum fator externo tendem a parasitar o mais rápido possível para assegurar a sobrevivência da progênie. Portanto, a combinação Bt $+\mathbf{T}$. pratissolii pode favorecer a atuação do parasitóide em campo, principalmente em casos em que é necessária uma rápida redução dos níveis populacionais da praga.

Palavras-chave: controle biológico, parasitóide de ovos, bioinseticida, MIP.

\begin{abstract}
This work was carried out to detect at laboratory the possible side-effects provoked by Bacillus thuringiensis (Bt) strains on egg parasitoid Thichogramma pratissolii and its progeny. Six strains of Bt E-3, E-10, E-15, E-16, E-19, E-20 and Bt kurstaki were assayed in honey drop to stimulate the feeding of the parasitoids in each treatment (1:1) and pure honey as check treatment and offered simultaneously displays with eggs of the host alternative Anagasta kuehniella. The experiment was maintained in a climatized chamber at $25 \pm$ $1^{\circ} \mathrm{C}, \mathrm{RH} 70 \pm 10 \%$ and photofase of 14 hours. The survival, daily parasitism, accumulated parasitism and total parasitism and also the progeny's emergence and sexual ratio were analyzed. Bt isolates did not affect parasitism, sexual ratio and longevity, but some of them (E-3, E-10, E-16 e E-20) affected parasitoid progeny. In field conditions this could imply in more parasitoid liberations to the expected results. Otherwise, parasitism acceleration verified in all treatments suggest that when T. pratissolii adults pass on some extern pressure (stress) they parasitize as soon as possible to assure progeny survival. The interaction $\mathbf{B t}+\mathbf{T}$. pratissolii may increase the parasitoid preformance in the field, mainly when a rapid decrease of pest population is desirable.
\end{abstract}

Key words: biological control, egg parasitoid, biopesticide, IPM.

\section{INTRODUÇÃO}

A bactéria Bacillus thuringiensis (Bt) destaca-se como entomopatógeno mais utilizado e

\footnotetext{
${ }^{1}$ Departamento de Fitotecnia, Universidade Federal do Espírito Santo (UFES), Centro de Ciências Agrárias, Alegre, ES, Brasil. ${ }^{2}$ Departamento de Fitotecnia, UFES, Centro de Ciências Agrárias, Alegre, ES. Brasil. E-mail: ricardo@cca.ufes.br, Alto Universitário s/n., Centro, 29500-000, Alegre, ES, Brasil. Autor para correspondência.

${ }^{3}$ Departamento de Biologia Animal, Setor de Entomologia, Universidade Federal de Viçosa (UFV), 36571-000, Viçosa, MG, Brasil.

${ }^{4}$ Departamento de Agronomia/Fitossanidade, Universidade Federal Rural de Pernambuco (UFRPE), 522171-900, Recife, PE, Brasil.

${ }^{5}$ Laboratório de Fitopatologia, Centro de Ciências Agrárias, UFES, 29500-000, Alegre, ES, Brasil.
} 
estudado no controle de insetos, sendo responsável por $90 \%$ do mercado mundial de bioinseticidas. Isolados de $\boldsymbol{B t}$ e/ou bioinseticidas à base desta bactéria têm ação patogênica contra mais de 1.000 espécies de insetos, destacando-se os lepidópteros com 572 espécies suscetíveis (POLANCZYK \& ALVES, 2003). Além disso, sua alta especificidade e seletividade favorecem a preservação do meio ambiente sendo uma grande vantagem para o agricultor, por outro lado, sua baixa persistência em campo é um dos principais obstáculos à sua utilização em larga escala (GLARE \& O’CALLAGHAM, 2000).

Apesar dos produtos à base de $\boldsymbol{B t}$ corresponderem a menos de $1 \%$ do mercado mundial de inseticidas, GLARE \& O'CALLAGHAM (2000) salientam a importância de estudos sobre o impacto ambiental deste entomopatógeno, visando principalmente a mostrar sua capacidade de substituir ou interagir com os inseticidas convencionais, minimizando os riscos ambientais. Os mesmos autores ressaltam que generalizações, nestes aspectos, são difíceis devido ao grande número de isolados existentes (mais de 60.000) e que cada caso deve ser analisado separadamente.

Entre os parasitóides, as espécies de Trichogramma (Hymenoptera, Trichogrammatidae) são, sem dúvida, as mais estudadas e utilizadas em todo o mundo, atualmente, pela sua eficiência e facilidade de criação em laboratório. Com relação ao Brasil, sua importância é relevante devido ao potencial de controle de pragas em diversas culturas (PINTO, 1997). Sua utilização deve-se ao fato de que esse microhimenóptero foi relatado parasitando mais de 200 espécies, pertencentes a 70 famílias de 8 ordens de insetos (PRATISSOLI \& PARRA, 2001), e em mais de 30 países, contra pragas chaves de 34 culturas, sendo liberados, de forma inundativa, em cerca de 15 milhões de hectares (Van LENTEREN \& BUENO, 2003). Países como Rússia, China, Alemanha, França, México e Suíça têm produzido e liberado Trichogramma spp. em extensas áreas, visando ao controle de diversas pragas.

A interação desses dois agentes de controle é comum em programas de Manejo Integrado de Pragas, como, por exemplo, na cultura do tomate no México, Colômbia e Brasil (TRUMBLE \& ALVARADORODRIGUEZ, 1993; HAJI et al., 2002). Embora os efeitos prejudiciais dos bioinseticidas à base de $\boldsymbol{B} \boldsymbol{t}$ sobre os inimigos naturais sejam mínimos e/ou significativamente menores que os dos agrotóxicos (GLARE \& O’CALLAGHAM, 2000), esses não podem ser desprezados e estudos são necessários em regiões onde essas táticas são empregadas em conjunto ou têm potencial de uso.

Este trabalho foi realizado com o objetivo de determinar o efeito de $\boldsymbol{B} \boldsymbol{t}$ sobre fêmeas adultas de Trichogramma pratissolii Querino \& Zucchi (Hymenoptera, Trichogrammatidae) e sua progênie.

\section{MATERIAL E MÉTODOS}

O trabalho foi desenvolvido no Laboratório de Entomologia e Fitopatologia do Centro de Ciências Agrárias (CCA) da UFES, em Alegre, ES, utilizando-se os isolados de $\boldsymbol{B} \boldsymbol{t}$ E-3, E-10, E-15, E-16, E-19, E-20 e o Bt kurstaki (Dipel) e o parasitóide Trichogramma pratissolii, criado no Laboratório de Entomologia do CCA-UFES.

Os isolados de $\boldsymbol{B} \boldsymbol{t}$ foram cultivados em meio LB (extrato de levedura, 5,0g; peptona, 10g; cloreto de sódio, 5,0g; agar, 15g; em 1L de água destilada) durante 72 horas à temperatura de $28^{\circ} \mathrm{C}$. Após esse processo, as colônias foram transferidas para tubos Falcon com $5 \mathrm{~mL}$ de água destilada e esterilizada e centrifugados por 40 minutos por três vezes (5.000 rpm) para retirada do meio de cultura e toxinas indesejáveis. Após a última centrifugação, o material foi re-suspenso em água destilada esterilizada e utilizado no experimento. $\mathbf{O} \boldsymbol{B} \boldsymbol{t}$ kurstaki foi obtido de formulação comercial e utilizado conforme recomendação do fabricante.

A metodologia empregada na criação do hospedeiro alternativo (Anagasta kuehniella) foi a desenvolvida por PARRA(1997), porém adaptada para as condições locais, utilizando-se uma dieta à base de farinha de trigo integral e milho (97\%) e levedo de cerveja (3\%). Para a criação do parasitóide, foram utilizados recipientes de vidro $(14$ x $7 \mathrm{~cm})$, colocandose na parede interna, gotículas de mel, para a alimentação dos adultos e fechados com filme plástico de PVC. Para a manutenção, foram oferecidos ovos do hospedeiro alternativo, colados em retângulos de cartolina azul celeste $(2,0$ x 8,0cm), através de goma arábica diluída a $10 \%$, e inviabilizados pela exposição à lâmpada germicida, por um período de 40 minutos (STEIN \& PARRA, 1987).

Para a execução do experimento, foram individualizadas, para cada tratamento (isolados E- E3, E-10, E-15, E-16, E-19, E-20 + Bt kurstaki), 20 fêmeas recém emergidas do parasitóide (20 repetições), em tubos de Duran, contendo uma gotícula de mel inoculado com diferentes isolados de $\boldsymbol{B} \boldsymbol{t}$ em suas paredes (proporção 1:1). A cada 24h, foi oferecida para cada fêmea uma cartela de cartolina azul celeste (2,5 x É 
0,5cm) contendo 40 ovos de A. kuehniella colados com goma arábica a $10 \%$ e inviabilizados pela exposição à lâmpada germicida por 40 minutos. Os tubos foram posteriormente fechados com filme plástico de PVC. Para a testemunha foi fornecida gotícula de mel sem $\boldsymbol{B}$.

O experimento foi mantido em câmaras climatizadas reguladas a temperaturas de $25 \pm 1^{\circ} \mathrm{C}$, umidade relativa de $70 \pm 10 \%$ e fotofase de 14 horas, até a emergência dos descendentes, quando foram avaliados os parasitismos diário, acumulado e total e a longevidade dos indivíduos.

O delineamento experimental utilizado foi o inteiramente casualizado, sendo os resultados, submetidos à análise de variância, e as médias comparadas pelo teste de Tukey, a 5\% de probabilidade de erro, no caso do parasitismo total. Os parasitismos diários e acumulados do parasitóide, emergência e a razão sexual da progênie foram comparados através de analise de regressão linear. A sobrevivência foi comparada pelo método de distribuição de Weibull (SGRILLO, 1982).

\section{RESULTADOS E DISCUSSÃO}

O parasitismo diário de T. pratissoli em ovos

de A. kuehniella não sofreu alterações devido à presença do $\boldsymbol{B} \boldsymbol{t}$ no alimento conforme pode ser observado pela tendência das linhas em todos os tratamentos (Figura 1). Esta tendência é reforçada pela Tabela 1 que mostra que os isolados de $\boldsymbol{B} \boldsymbol{t}$ não afetaram o número total de ovos parasitados. Com exceção dos isolados E-3, E-16 e testemunha que o parasitismo no primeiro dia ficou bem próximo de $40 \%$, para todos os outros tratamentos este se situou entre 20 e $40 \%$ (Figura 1), sofrendo um decréscimo gradual nos dias seguintes (Figura 2). O índice de $80 \%$ de parasitismo acumulado em ovos de A. kuehniella foi alcançado em torno de 3,5 dias nos isolados E-10, E-15, E-16 e Bt kurstaki, aos 4 dias no isolado E-20, aos 4,5 dias no isolado E-19, no quinto dia no isolado E-3 e no sexto dia para a testemunha (Figura 1). Porém, apesar dessa aceleração do parasitismo o número total de ovos parasitados não foi afetado (Tabela 1), demonstrando que, apesar de $\boldsymbol{T}$. pratissolii ter ovipositado mais cedo em alguns tratamentos, isso não resultou em um maior parasitismo ao longo do tempo.

A aceleração do parasitismo verificada para esse parasitóide é importante porque em todos os tratamentos foram necessários menos dias para os parasitóides atingirem $80 \%$ de parasitismo acumulado. Esses resultados sugerem que adultos de T. pratissolii quando submetidos à pressão de algum fator externo, neste caso $\boldsymbol{B} \boldsymbol{t}$, tendem a parasitar o mais rápido possível para assegurar a sobrevivência da progênie. Portanto, a combinação $\boldsymbol{B t}+\boldsymbol{T}$. pratissolii pode favorecer a atuação do parasitóide em campo, principalmente em casos que é necessário uma rápida redução dos níveis populacionais da praga.

A longevidade em todos os tratamentos mostrou a mesma tendência, pois a mortalidade seguiu uma distribuição normal não sendo observada queda acentuada como mostra a distribuição de Weibull (Figura 3).

A emergência dos parasitóides foi afetada pelos tratamentos E-3, E-10, E-16 e E-20, pois em todos estes casos inicialmente a emergência ficou acima de 85\%, número considerado ideal por NAVARRO (1998), porém houve drástica redução nos dias seguintes. Os tratamentos E-15, E-19 e Bt kurstaki afetaram a emergência dos parasitóides, porém somente nos primeiros dias, pois foi observado um acréscimo importante com o passar o tempo A testemunha apresentou emergência satisfatóra e constante ao longo do tempo (Figura 4).

A razão sexual, de um modo geral, apresentou tendência de queda ao longo do tempo (Figura 5), porém a influência dos tratamentos não foi suficiente para reduzir os valores para abaixo de 0,5 , valor mínimo satisfatório para a razão sexual, conforme NAVARRO(1988).

Tabela 1 - Número de total de ovos de Anagasta kuehniella (Lepidoptera, Pyralidae) parasitados por Trichogramma pratissolii (Hymenoptera, Trichogrammatidae) alimentado com mel e Bacillus thuringiensis. $25 \pm 1^{\circ} \mathrm{C}$, UR $70 \pm 10 \%$ fotofase $14 \mathrm{~h}$.

Isolados ou subespécie de Número total de ovos parasitados ${ }^{1}$ Bacillus thuringiensis

\begin{tabular}{ll}
\hline E-3 & $31,39 \pm 1,92 \mathrm{~A}$ \\
E-10 & $34,94 \pm 2,99 \mathrm{~A}$ \\
E-15 & $24,50 \pm 2,59 \mathrm{~A}$ \\
& \\
E-16 & $22,66 \pm 2,12 \mathrm{~A}$ \\
E-19 & $23,27 \pm 2,88 \mathrm{~A}$ \\
E-20 & $29,83 \pm 2,09 \mathrm{~A}$ \\
Bt kurstaki & $33,56 \pm 1,98 \mathrm{~A}$ \\
Testemunha & $32,33 \pm 2,92 \mathrm{~A}$ \\
\hline
\end{tabular}

${ }^{1}$ Médias não seguidas pela mesma maiúscula diferem entre si pelo teste de Tukey a 5\% de significância.

$\mathrm{CV}=35,00 \%$

$\mathrm{n}=160$

Ciência Rural, v.36, n.2, mar-abr, 2006. 


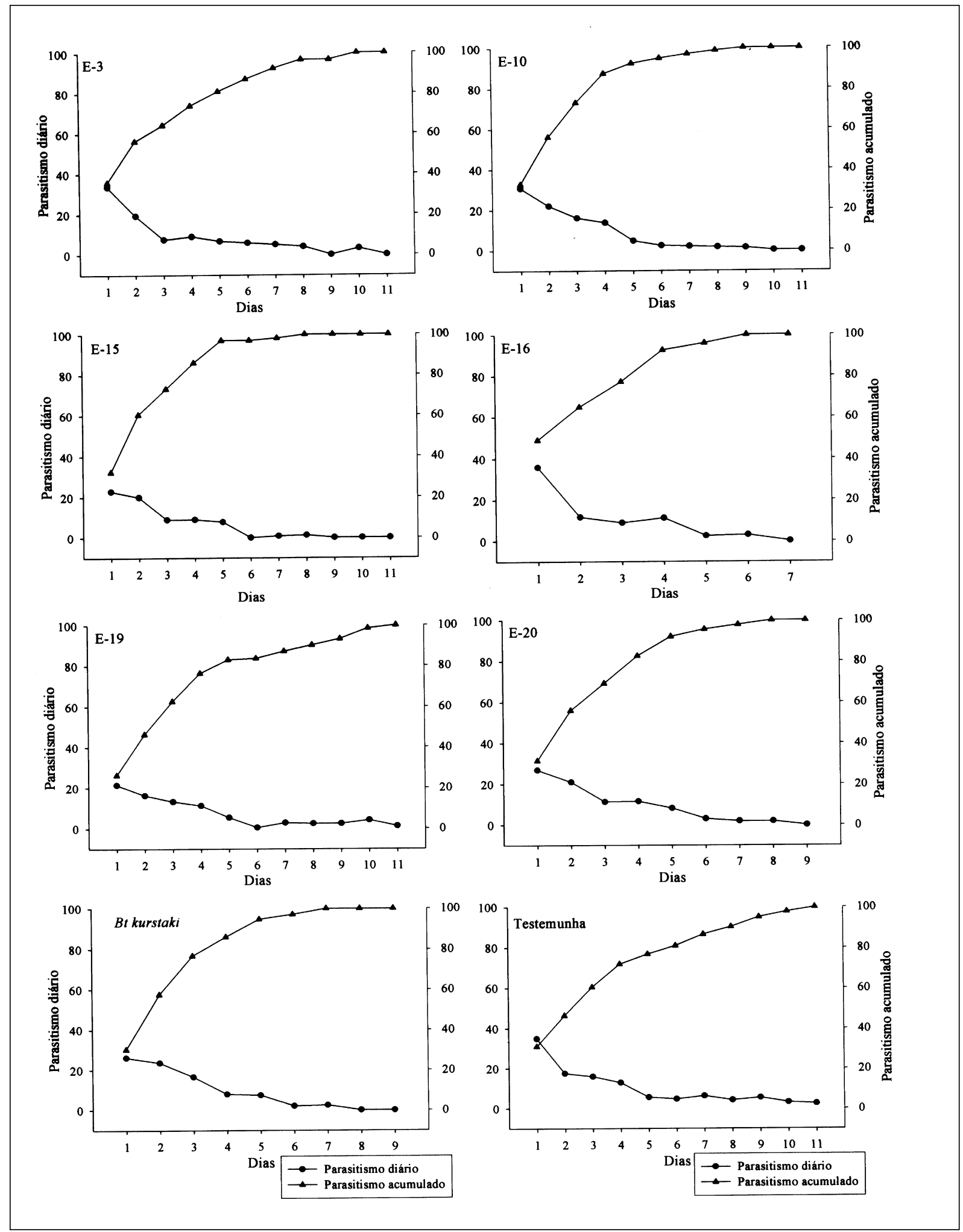

Figura 1 - Parasitismo diário e acumulado de Trichogramma pratissolii (Hymenoptera, Trichogrammatidae) alimentado com mel e diferentes isolados de Bacillus thuringiensis e Bt kurstaki.

Ciência Rural, v.36, n.2, mar-abr, 2006. 


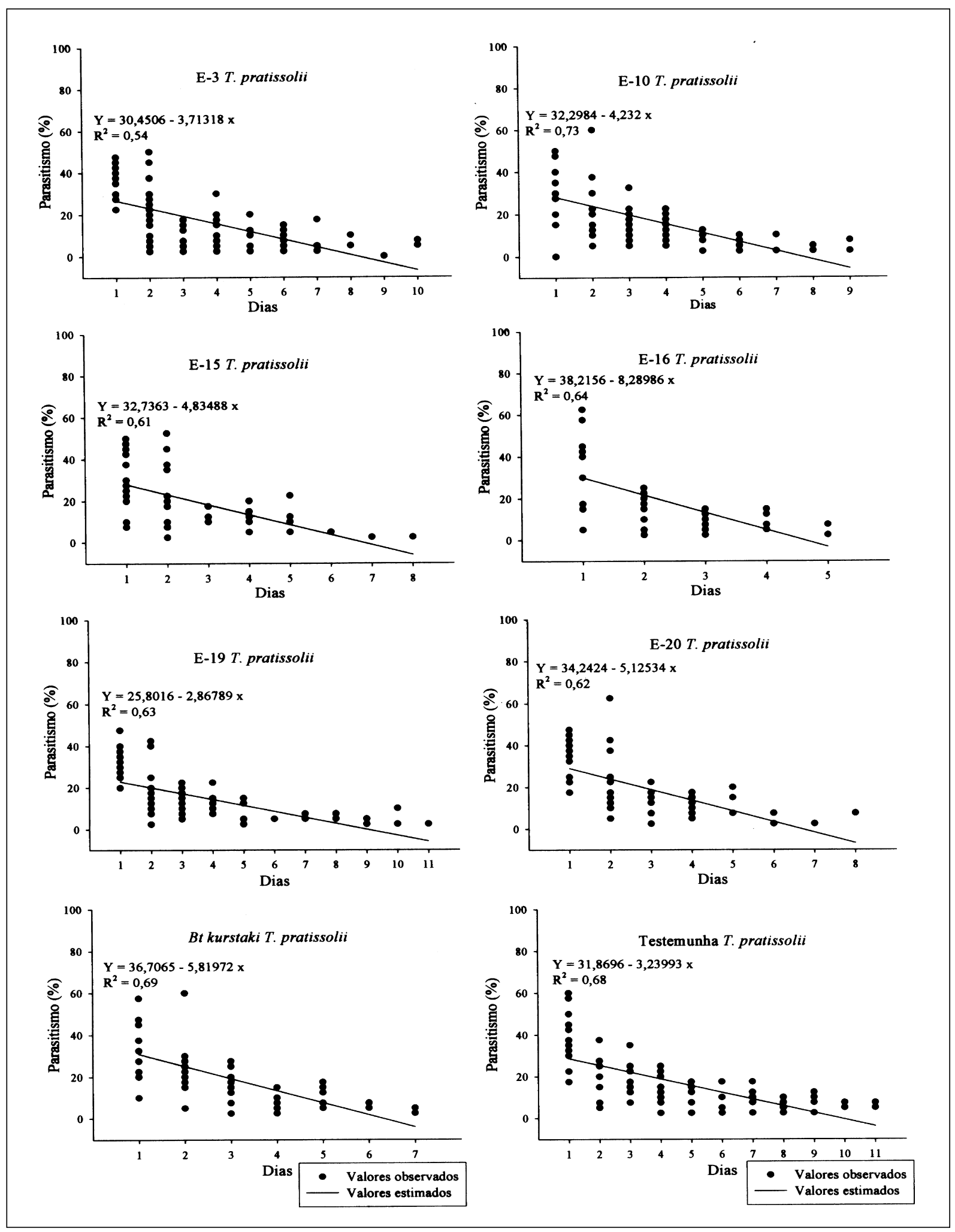

Figura 2 - Parasitismo de Trichogramma pratissolii (Hymenoptera, Trichogrammatidae) alimentado com mel e diferentes isolados de Bt e Bt kurstaki. 


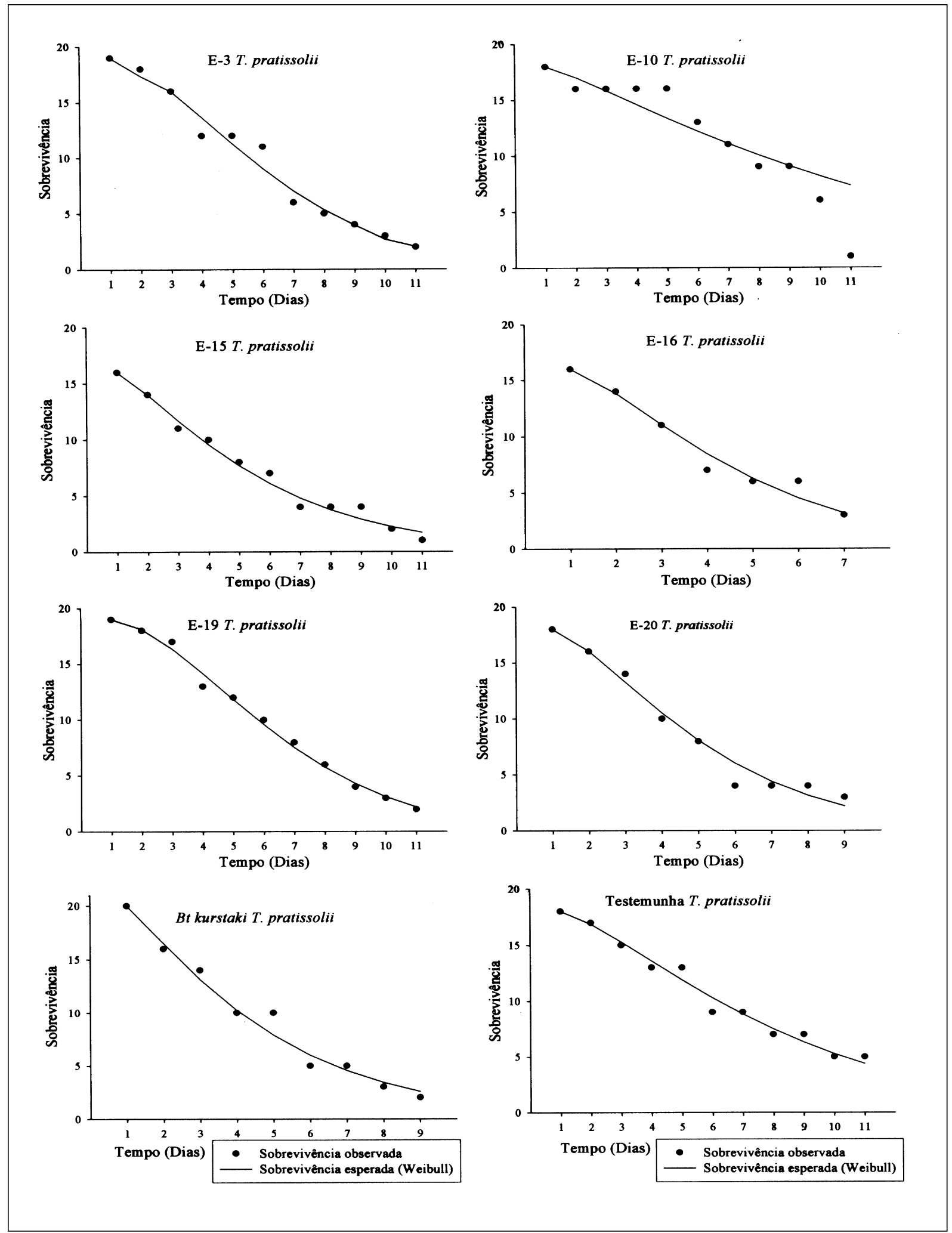

Figura 3 - Sobrevivência de Trichogramma pratissolii (Hymenoptera, Trichogrammatidae) alimentado com mel e diferentes isolados de $\boldsymbol{B} t$ e Bt kurstaki.

Ciência Rural, v.36, n.2, mar-abr, 2006. 

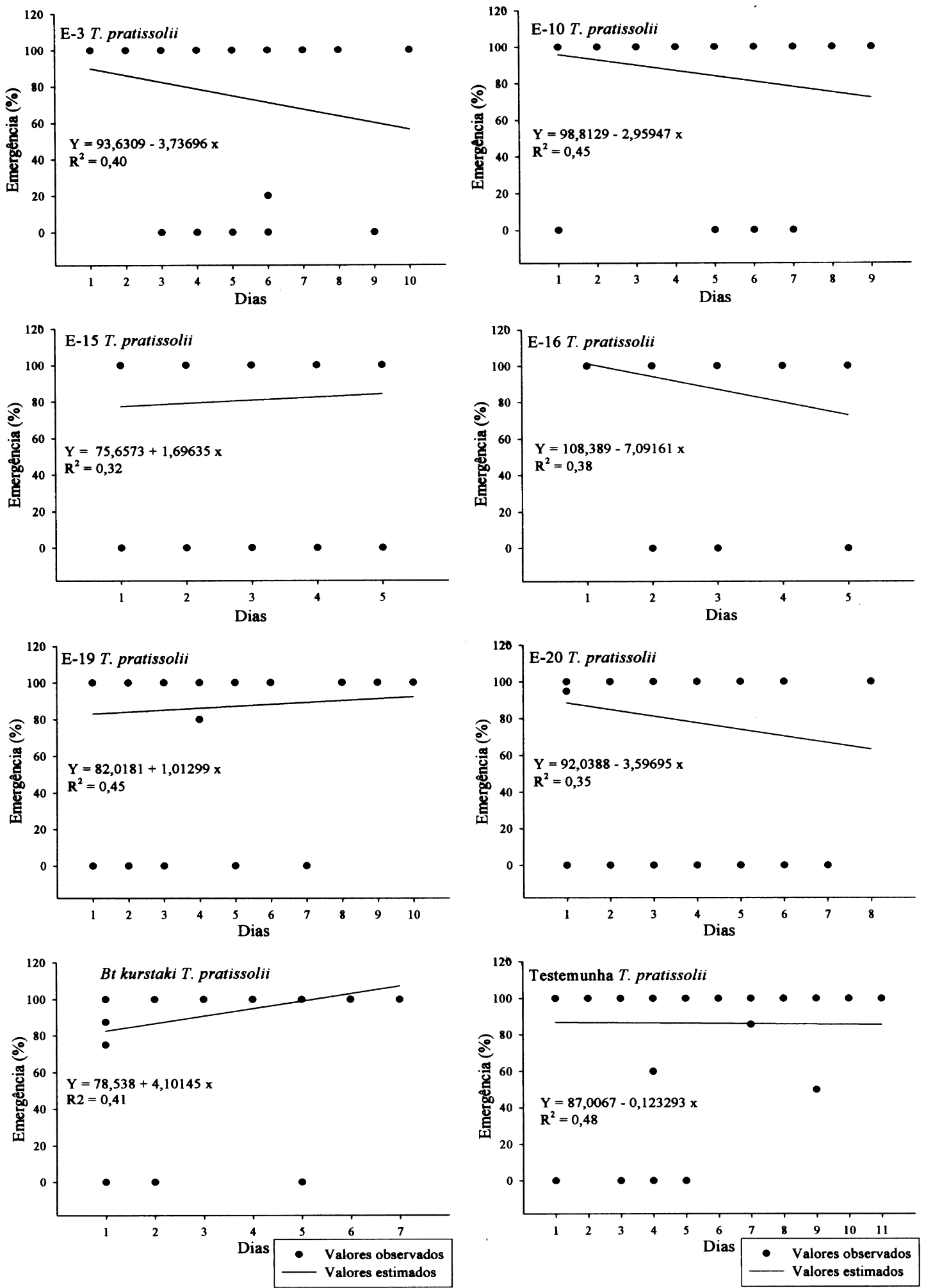

Figura 4 - Emergência (\%) de Trichogramma pratissolii (Hymenoptera, Trichogrammatidae) alimentado com mel e diferentes isolados de Bt e Bt kurstaki.

Ciência Rural, v.36, n.2, mar-abr, 2006. 

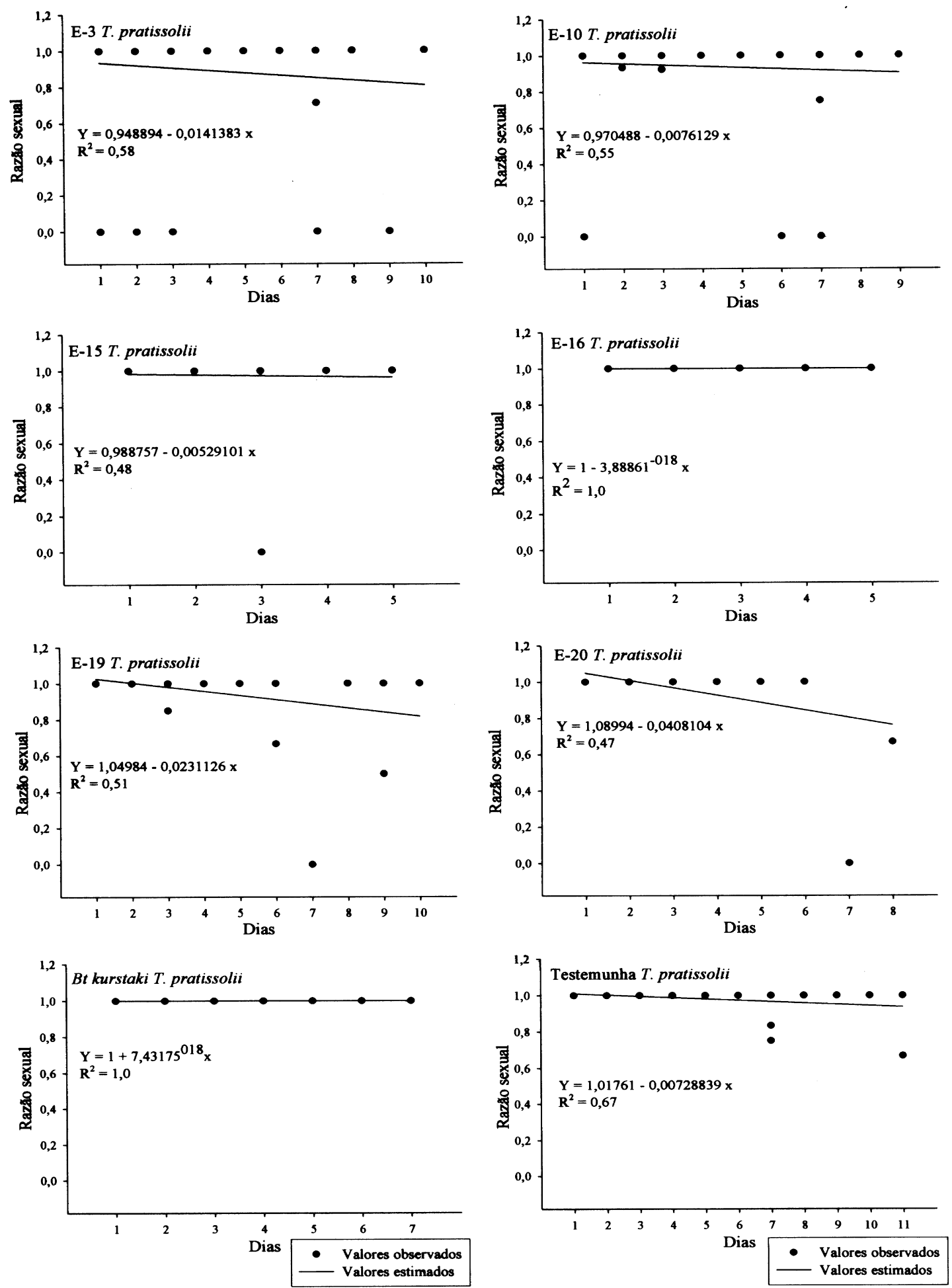

Figura 5 - Razão sexual de Trichogramma pratissolii (Hymenoptera, Trichogrammatidae) alimentado com mel e diferentes isolados de Bt e Bt kurstaki. 
É importante ressaltar que a subespécie Bt kurstaki é a base da maioria dos bioinseticidas formulados com Bt (GLARE \& O’CALLAGHAM, 2000) e, que, neste estudo, foi verificado que este tratamento não afetou os parâmetros estudados (Figuras 1,2,3,4 e 5 e Tabela 1), confirmando a viabilidade da interação entre $\boldsymbol{B t}$ kurstaki e Trichogramma em campo, verificada por KOGAN et al.(1998) e HAJI et al. (2002).

Este trabalho mostra que $\boldsymbol{B t}$ fornecido via alimento para adultos de $\mathbf{T}$. pratissolii não afeta o parasitismo. Porém, em alguns tratamentos (E-3, E-10, E-16 e E-20) foi observado efeito indireto sobre a emergência da progênie. Esses isolados não são recomendados para futuros estudos de interação $\boldsymbol{B} \boldsymbol{t}$ e Trichogramma, pois seu emprego implicaria a necessidade de mais liberações do parasitóide devido ao $\boldsymbol{B} \boldsymbol{t}$ afetar a quantidade (emergência) da progênie dos parasitóides.

\section{CONCLUSÕES}

Bacillus thuringiensis não afeta o parasitismo e a sobrevivência de Trichogramma. pratissolii. Os isolados E-3, E-10, E-19 e E-20 afetam a emergência do Trichogramma pratissolii.

\section{REFERÊNCIAS}

GLARE, T.R.; O’CALLAGHAM.M. Bacillus thuringiensis: biology, ecology and safety. Chichester: John Wiley \& Sons, 2000. 350p.

HAJI, F.N.P. et al. Trichogramma pretiosum para o controle de pragas no tomateiro. In: PARRA, J.R.P. et al. Controle biológico no Brasil, parasitóides e predadores. São Paulo: Manole, 2002. Cap.28, p.477-494.
KOGAN, M. Integrated pest management: historical perspectives and contemporary development. Annual Review of Entomology, Palo Alto, v.43, p.243-270, 1998.

NAVARRO, M.A. Trichogramma spp. Producción, uso y manejo em Colômbia. Guadalajara de Buga: Impretec, 1998. $176 p$.

PARRA, J.R.P. Técnicas de criação de Anagasta huehniella, hospedeiro alternativo para produção de Trichogramma. In: PARRA., J.R.P.; ZUCCHI, R.A. Trichogramma e o controle biológico aplicado. Piracicaba: FEALQ, 1997. Cap.4, p.121150 .

PINTO, J.D. Taxonomia de Trichogrammatidae (Hymenoptera) com ênfase nos gêneros que parasitam Lepidoptera. In: PARRA, J.R.P.; ZUCCHI, R.A. Trichogramma e o controle biológico aplicado. Piracicaba: FEALQ, 1997. Cap.1, p.13-40.

POLANCZYK, R.; ALVES, S. Bacillus thuringiensis: uma breve revisão. Agrociência, Montevidéu, v.7, p.1-10, 2003.

PRATISSOLI, D.; PARRA, J.R.P. Seleção de linhagens de Trichogramma pretiosum Riley (Hymenoptera: Trichogrammatidae) para o controle das traças Tuta absoluta (Meyrick) e Phthorimaea operculella (Zeller) (Lepidoptera: Gelechiidade). Neotropical Entomology, Londrina, v.30, p.277-282, 2001.

SGRILLO, R.B. A distribuição de Weibull como modelo de sobrevivência de insetos. Ecossistema, Espírito Santo do Pinhal, v.7, p.9-13, 1982.

STEIN, C.P.; PARRA. J.R.P. Uso da radiação para inviabilizar ovos de Anagasta kuehniella (Zeller, 1879) visando estudos com Trichogramma spp. Anais da Sociedade Entomológica do Brasil, Sete Lagoas, v.16, p.229-231, 1987.

TRUMBLE, J.; ALVARADO-RODRIGUEZ. B. Development of economic evaluation of an IPM program for fresh market tomato production in Mexico. Agriculture, Ecosystems \& Environment, Charlottetown, v.43, p.267-284, 1993

Van LENTEREN, J.C.; BUENO, V.H.P. Augmentative biological control of arthropods in Latin America. BioControl, Helsinki, v.48, p.123-138, 2003. 